\title{
The dangers of attacking disease programmes for developing countries
}

\author{
In this Observations paper (BMJ 2007;335:646, doi:10.1136/ \\ bmj.39349.591296.59), an author's name was misspelt. 'Gregg \\ Gonsales' should be 'Gregg Gonsalves'.
}

